\title{
STUDY OF THE EFFECT OF LOW-TEMPERATURE PLASMA EXPOSURE ON MOULD FUNGI COLONIZING PAPER
}

\author{
Gontcharova I. ${ }^{a, *}$, Arashkova A. ${ }^{a}$, Bordusau S. ${ }^{b}$, Madveika S. ${ }^{b}$, \\ Filatova I. ${ }^{c}$, Lyushkevich V. ${ }^{c}$, Brablec A. ${ }^{d}$ \\ ${ }^{a}$ Institute of Microbiology of the National Academy of Sciences of Belarus, Kuprevich str. 2, 220014 Minsk, \\ Belarus \\ ${ }^{b}$ Belarusian State University of Informatics and Radioelectronics, P.Brovki 6, 220013 Minsk, Belarus \\ ${ }^{c}$ B.I. Stepanov Institute of Physics of the National Academy of Sciences of Belarus, Nezavisimosti ave. 70, \\ 220072 Minsk, Belarus \\ ${ }^{d}$ Masaryk University, Faculty of Science, Department of Physical Electronics, Kotlarska 2, 61137 Brno, Czech \\ Republic \\ * sorbic@mbio.bas-net.by
}

\begin{abstract}
The influence of radiofrequency cold plasma in ambient air at $200 \mathrm{~Pa}$ pressure on mould fungi of the genus Aspergillus was studied. It was shown that 20 min plasma treatment of paper with mould and contamination reduced the number of colony forming units but didn't lead to complete suppression of fungal viability. Strains of A.versicolor after plasma treatment lost the ability to excrete pink pigment to the environment. A.niger strains were more resistant and kept acidification ability. One of the possible plasma inactivation factors was formation of reactive hydroxyl $(\mathrm{OH})$ radical.
\end{abstract}

Keywords: RF cold plasma, mould fungi Aspergillus, emission spectrum.

\section{Introduction}

Colonization of paper by microscopic (mould) fungi is rather common in archives, libraries, museums. The most visible effect of paper biodeterioration are stains due to colored fungal spores or exopigments. Mould fungi may cause serious damage in materials, which are decomposed from the impact of enzymes and organic acids [1].

Librarians and archivists who work with old books and documents are exposed to fungal spores some of which are potentially dangerous for human health. Mould spores flying in the air can provoke allergic reactions [2. Professional diseases among librarians may be mycoses of lungs and skin. Fungi belonged to the genus Aspergillus, characterized by high level organic acids synthesis and a substantial tolerance to environmental conditions, are particularly hazardous. They are causative agents of a large spectrum of diseases known as aspergillosis [3].

The removal of microbial contamination from old books is a serious problem. Mechanical cleaning is laborious and ineffective because fungal hyphae can penetrate into paper porous forming mycelium netting. The chemical treatment using biocides usually has a negative impact on paper object safety [1].

A new technology in the field of paper conservation has been recently proposed. It is based on plasma treatment technique, which is widely used in a number of other applications. After plasma treatment, no harmful residues remain in treated materials. High frequency $(\mathrm{HF})$ cold plasma has been studied to elaborate a non-destructive method for cleaning and decon- tamination of cultural heritage objects. With regard to paper, the main advantages of plasma treatment are the dry nature of the process and the possibility of combining different effects, such as elimination of microbes and strengthening of deteriorated materials through the deposition of superficial layers [4]. The removal of different sediments from contaminated surface with treatment in oxygen/argon HF plasma is one of the various possible ways of plasma application [5].

Significant advance has been made in cold plasma treatment of the simplest prokaryotic microorganisms. But in the last decades the opportunity to use low-temperature plasma for sterilization of paper contaminated with multicellular fungal organisms was investigated [6].

The aim of the investigation was to study the effect of treatment of mould fungi colonizing paper by lowpressure cold plasma of radiofrequency discharge.

\section{Experimental}

Treatment was carried out in a planar geometry capacitively coupled radiofrequency $(\mathrm{RF})$ plasma reactor consisting of two plane-parallel water-cooled copper electrodes placed in a stainless steel vacuum chamber with the internal volume of $0.053 \mathrm{~m}^{3}$. The distance between electrodes was $20 \mathrm{~mm}$. One of the electrodes was connected to $5.28 \mathrm{MHz}$ generator (VNIITVCH, Russia), and the other one was grounded. Specimens were put on the grounded electrode. The treatments were performed in ambient air at a pressure of $200 \mathrm{~Pa}$ and a specific power density varied in the range of 

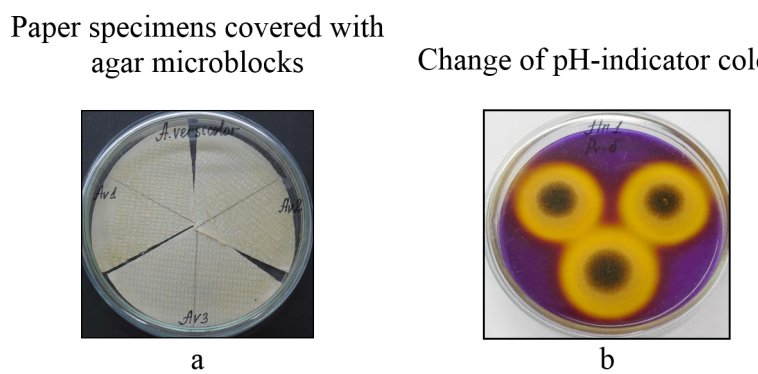

Excretion of pigments

Figure 1. Agar microblocks inoculated with test-culture spores on paper specimens (a), medium with bromocresol purple (b) pigmentation of medium (c).

\begin{tabular}{c|c|c}
\hline \hline \multirow{2}{*}{ Culture } & \multicolumn{2}{|c}{ CFU $/ \mathbf{c m}^{2}$} \\
\cline { 2 - 3 } & Control & 20 min plasma \\
\hline A.niger A49 & $4.0-7.8 \times 10^{6}$ & $2.9-7.6 \times 10^{4}$ \\
A.niger A70 & $2.6-3.3 \times 10^{7}$ & $1.9-2.8 \times 10^{6}$ \\
A.niger M19 & $4.7-9.2 \times 10^{6}$ & $2.3-8.5 \times 10^{5}$ \\
A.versicolor A75 & $1.6-3.4 \times 10^{6}$ & $5.6-7.3 \times 10^{3}$ \\
A.versicolor M22 & $3.5-5.3 \times 10^{5}$ & $5.8-8.0 \times 10^{2}$ \\
A.versicolor HXM17 & $5.8-8.0 \times 10^{5}$ & $2.8-4.2 \times 10^{3}$ \\
\hline \hline
\end{tabular}

Table 1. Number of CFU of A.niger and A.versicolor strains treated with low-temperature plasma.

$0.3-0.5 \mathrm{~W} / \mathrm{cm}^{3}$. The duration of plasma exposure was $20 \mathrm{~min}$.

Spectral composition and thermodynamic parameters of plasma were investigated by optical emission spectroscopic methods using MS3504 series monochromator-spectrograph (SOL instruments). Emission spectra were recorded in the wavelengths range of $250-1100 \mathrm{~nm}$.

Spectra of the $\mathrm{N}_{2}$ 2nd positive system enabled to determine rotational $T_{r}$ and vibrational $T_{v}$ temperatures by fitting the experimental spectra with the simulated ones. For spectral simulation SPECAIR Version 2.1 software was used.

The anti-microbial efficacy of non-thermal plasma treatment was tested on the microscopic (mould) fungi belonging to the genus Aspergillus. Species A.niger and A.versicolor were chosen as the most dangerous deterioration agents of paper documents and books due to the ability of organic acids and pigment production.

For mould contamination of paper specimens spore suspension was prepared by pouring $3 \mathrm{ml}$ sterile water into the glass tubes with Aspergillus culture and scraping the surface of agar medium with microbiological wire loop. The obtained spore suspension was mixed with mild nutrient medium (Czapek-Dox agar) and spread on paper using mesh pattern so that the microblocks of the same size sticked to the surface that modeled organic contamination (Fig. 1). Inoculated paper specimens were incubated for 3 days at $28^{\circ} \mathrm{C}$ for mycelium network formation and spores generation. After plasma treatment spores were washed and $100 \mu \mathrm{l}$ from each of the serial dilution was spread on Czapek-Dox agar plates. The number of colony forming units (CFU) was counted after 3 days of cultivation. Unexposed to plasma treatment specimens were used for evaluation of concentration of CFU in control.

For investigation of organic acid production by fungi (acidification) agar microblocks were placed in Petri dishes by 3-point inoculation of nutrient medium with bromocresol purple as $\mathrm{pH}$ indicator. Yellow area around fungal colony was measured as acidification zone. Pigment excretion by fungi was estimated visually according to the medium staining intensity (Fig. 1).

\section{Results}

The examination of about 400 probes of dust from 8 archives, libraries and museums in Minsk showed that the most dangerous Aspergillus species A. niger and $A$. versicolor were common among mould fungi on paper objects.

Investigation of 20 minutes exposure to RF discharge cold plasma at low-pressure on Aspergillus colonizing paper showed the ingibition of mycelium development of all tested cultures. The survival rate varied not only at the species level (A.niger and A.versicolor) but was strain specific. In general the number of A.versicolor $\mathrm{CFU}$ on plasma treated paper decreased to a greater extent than of A.niger strains (table 1).

It was found that the growth rate of $A$.niger colonies formed from treated agar microblocks decreased insignificantly. Maximum difference in colony diameter between plasma treated and control variants was $5 \mathrm{~mm}$.

The damage ability of fungi is different. A.niger 


\begin{tabular}{c|c|c|c|c}
\hline \hline \multirow{2}{*}{ Fungus } & \multicolumn{2}{|c|}{ Without plasma treatment } & \multicolumn{2}{|c}{ After plasma treatment } \\
\cline { 2 - 5 } & $\begin{array}{c}\text { Colony } \\
\text { diameter, mm }\end{array}$ & $\begin{array}{c}\text { Acidification } \\
\text { zone, mm }\end{array}$ & $\begin{array}{c}\text { Colony } \\
\text { diameter, mm }\end{array}$ & $\begin{array}{c}\text { Acidification } \\
\text { zone, mm }\end{array}$ \\
\hline A.niger A49 & $33-34$ & $8-9$ & $27-31$ & $1-2$ \\
A.niger A70 & $32-33$ & $7-8$ & $30-31$ & $4-8$ \\
A.niger M19 & $26-33$ & $7-8$ & $29-30$ & $10-11$ \\
\hline \hline
\end{tabular}

Table 2. Influence of plasma treatment on A.niger strains growth and organic acids production in 3 days cultivation on Czapek-Dox medium with bromocresol purple.

\begin{tabular}{c|c|c|c|c}
\hline \hline \multirow{2}{*}{ Fungus } & \multicolumn{2}{|c|}{ Without plasma treatment } & \multicolumn{2}{c}{ After plasma treatment } \\
\cline { 2 - 5 } & $\begin{array}{c}\text { Colony } \\
\text { diameter, mm }\end{array}$ & $\begin{array}{c}\text { Pigmentation } \\
\text { zone }^{*}\end{array}$ & $\begin{array}{c}\text { Colony } \\
\text { diameter, mm }\end{array}$ & $\begin{array}{c}\text { Pigmentation } \\
\text { zone * }\end{array}$ \\
\hline A.versicolor A75 & $9-12$ & +++ & $3-4$ & - \\
A.versicolor M22 & $9-13$ & ++ & $4-5$ & - \\
A.versicolor HXM17 & $11-14$ & + & $7-8$ & - \\
\hline \hline
\end{tabular}

Table 3. Influence of plasma treatment on A.versicolor strains growth and pigment excretion in 6 days cultivation on Czapek-Dox medium.

*Intensity level: - complete absence, + low, ++ average, +++ high.

causes discolouration of pictures and deterioration of paper producing organic acids - aggressive metabolites causing chemical destruction. A.versicolor excretes bright pigments of all shades of pink.

The presence of yellow zones around A.niger colonies indicated that all A.niger strains after plasma treatment preserved the ability to produce organic acids. But if A.niger A49 acidification zones decreased significantly then A.niger M19 ones were even wider than in control. Obtained resulted pointed out that plasma exposure can influence on fungal aggressiveness (table 2). In case of A.versicolor, strains were more sensitive to plasma sterilization process. Plasma slowed down fungal growth: colonies were 2-5 times less than in control, excretion of pigment to the environment ceased (table 3).

When inoculated paper specimens were placed in vacuum chamber $(200 \mathrm{~Pa})$ for 20 minutes without $\mathrm{RF}$ discharge plasma the number of $\mathrm{CFU}$ and growth parameters of fungi didn't differ from the control.

The composition of the emission spectra gives an insight into the ongoing plasma chemistry. Molecular $(\mathrm{OH})$ radical, and other active species, $N_{2}(\mathrm{C}, \mathrm{A})$, $N_{2}^{+}$(B), were identified (Fig. 2). The temperatures measured in RF discharge plasma were the following: vibrational temperature $T_{v}=3000 \mathrm{~K}$, gas temperature $T_{g}$ (equals with $T_{r}$ ) was $320 \mathrm{~K}$. The difference in $T_{v}$ and $T_{r}$ values indicates the non-equilibrium in lowpressure RF plasma and gives evidence that the gas temperature cannot be among plasma inactivation factors. This fact ensures non destructive treatment of thermosensitive biopolymeric material (paper).

We consider that possible explanation of plasma toxic effect lies in formation of hydroxyl $(\mathrm{OH})$ radical which is known as one of the most reactive species of biological importance [6].

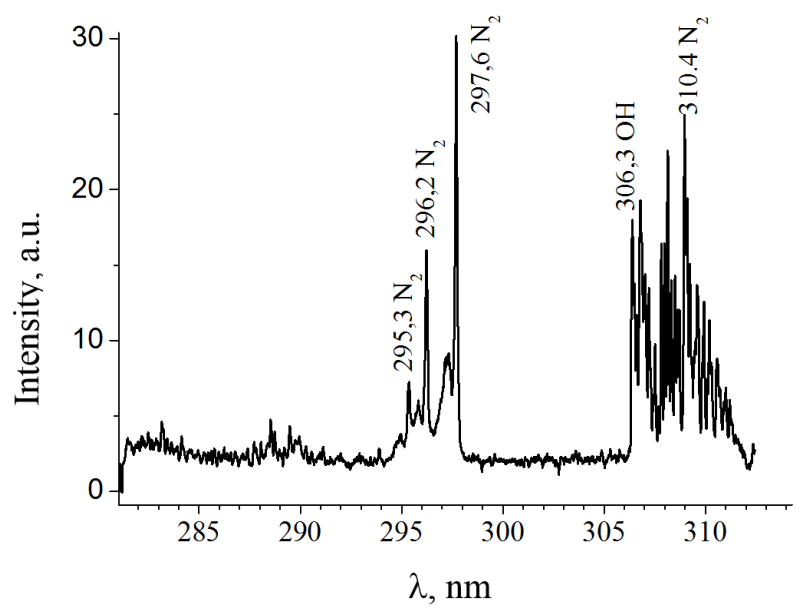

Figure 2. Emission spectrum of RF plasma in 280-340 $\mathrm{nm}$ region.

\section{Conclusions}

The resistance of mould fungi A.niger and A.versicolor to low-temperature plasma is comparatively high. The treatment with cold plasma of paper specimens contaminated with fungal mycelium results in the reduction of colony forming units. However complete suppression of hypha viability did not occur even when exposure continued 20 minutes. After plasma treatment A.versicolor lost the ability to excrete pink pigment to the environment. A.niger was more resistant to external influence retaining the acid-producing ability.

\section{Acknowledgements}

This research has been supported by the Belarusian Republican Foundation for Fundamental Research (grant T15MC-050). 


\section{References}

[1] Silvia O. Sequeira Eurico J. Cabrita Maria F. Macedo. Fungal biodeterioration of paper: How are paper and book conservators dealing with it? Restaur, 35(2):188-199, 2014.

[2] Green B.J. Mitakakis T.Z. Tovey E.R. Allergen detection from 11 fungal species before and after germination. J. Allergy Clin. Immunol, 111(2):285-289, 2003.

[3] Segal B.H. Walsh T.J. Current approaches to diagnosis and treatment of invasive aspergillosis. J.Respir. Crit. CareMed., 173:707-717, 2006.

[4] Laguardia L. Vassallo E. Cappitelli F. Mesto E. Cremona A. Sorlini C. Bonizzoni G. Investigation of the effects of plasma treatments on biodeteriorated ancient paper. Applied Surface Science, 252:1159-1166, 2005.

[5] Totolin M. Macocinschi D. Ioanid G. E. Filip D. Ioanid A. Materials supports for cultural heritage objects treated in cold plasma. Optoelectronics and advanced materials-rapid communications, 1(12):722-728, 2007.

[6] Vrajova J. Chalupova L. Cech J. Krcma F. Stahel P. Paper sterilization by atmospheric pressure dbd discharge. Chem. Listy, 102:1445-1449, 2008. 